\title{
NEWS
}

\section{Green money laundering}

\author{
John A. Glaser
}

Published online: 11 April 2014

(C) Springer-Verlag Berlin Heidelberg (outside the USA) 2014

New banknotes are printed to replace worn or unfit currency each year across the world. The central banks responsible for the currency printing are faced with the environmental challenge of annually disposing of nearly 150,000 tons of notes unfit for recirculation. Banknote soiling has been found to be a yellowing of the notes due to the accumulation of oxidized sebum, a secretion of the sebaceous glands in the human skin. The oily/waxy sebum exudate lubricates the human skin and hair as a natural process. Studies by the De Nederlandsche Bank identified yellowing of the banknotes attributable to the air oxidation of sebum. This contamination leads to mechanical problems with the notes in high speed banknotecounting machines.

Supercritical $\mathrm{CO}_{2}\left(\mathrm{SCCO}_{2}\right)$ has been shown to remove sebum, other oils, and contaminants, including common bacterial colonies, from both paper and polymer banknotes without destroying the costly and sophisticated security features. $\mathrm{SCCO}_{2}$ cleaning at $60{ }^{\circ} \mathrm{C}$ and $5,000 \mathrm{psi}$ was shown to be effective to clean conventional straps of 100 banknotes, extracting nearly $4 \%$ of the initial strap weight. Measurements of note-soiling distributions on a banknotesorting machine running at 10 banknotes per second showed a significant shift in soiling levels after cleaning, supporting the claim that processing of $\mathrm{SCCO}_{2}$ cleaned notes would result in significantly fewer notes being classified as unfit due to soiling and shredded for disposal. Soiling accounts for the removal of $60-80 \%$ of all banknotes. Effective cleaning of banknotes could decrease the

J. A. Glaser ( $\square)$

US Environmental Protection Agency, National Risk

Management Research Laboratory, 26 W King Dr, Cincinnati, OH 45268, USA

e-mail: Glaser.John@epa.gov annual global cost of replacing the bills now approaching $\$ 10$ billion.

Ind Eng Chem Res 2014, 53, 530-540

\section{Route-scouting analysis using footprint quantification}

The pharmaceutical industry has shown exceptional interest in the sustainability of process chemistries currently in use and where future needs lie. The ACS Green Chemistry Institute $^{\circledR}$ Pharmaceutical Roundtable (ACS GCI ${ }^{\circledR}$ PR) initiative has provided a meeting of minds scenario for the investigation of the sustainability of pharmaceutical chemistry. The sustainability or greenness of a chemical process has been the object of various univariate metrics such as the E-factor. Defined as the actual waste generated during the production of the desired product ( $\mathrm{kg}$ waste $/ \mathrm{kg}$ final product), the E-factor takes into account the process yield with accompanying reagents and solvents. Incorporation of water in the process can obscure meaningful comparisons due to exceptionally high E-factors. ACS $\mathrm{GCI}^{\circledR} \mathrm{PR}$ selected process mass intensity (PMI) as the metric of choice which allows competing process data to be compared on a transparent and equitable basis. The PMI is composed of raw material quantities used in the production of the desired product ( $\mathrm{kg}$ raw materials $/ \mathrm{kg}$ product). All materials used directly in the synthetic process, isolation, and purification of the product are considered raw materials. PMI is easily calculated but does not have weighting factors of the individual raw materials which would be a more useful sustainability metric.

$\mathbf{P M I}_{\text {product }}=\frac{\text { total mass of process or process step }(\mathrm{kg})}{\text { mass of product }(\mathrm{kg}) \text { for process or process step }}$ 
In practice, different synthetic routes are compared and ranked for their sustainability performance as an essential step toward the development of chemical processes that strive to be benign by design. This report offers a methodology proposed to enable process chemists at an early route-scouting phase of a targeted process to estimate the carbon footprint of different synthetic route options. Carbon footprint (CFP) "corresponds to the total greenhouse emissions caused directly and indirectly by the production/ isolation of the raw material, would be a suitable sustainability weighting factor." For common solvent and bulk chemicals, the footprint data are readily available to process chemists, and the CFP can be calculated using:

$\mathbf{C F P}_{\text {product }}=\frac{\sum(\mathrm{kg} \text { specific material }) \times(\mathrm{CFP} \text { raw material })}{\text { final product }(\mathrm{kg})}$

The CFP (with included calculation schemes) can be used to evaluate a total product footprint through the inclusion of the energy contribution to the overall carbon footprint, using a rule of thumb for zero-order estimation of the carbon footprint of raw materials, and implication of flexible front gate loading. This simple approach to product carbon footprint determination can assist the process chemists at early stages of synthetic route selection. The intercomparison of synthetic routes offer the ability to rank route options on a sustainability basis.

Chimica Oggi-Chem Today 2013, 31(6), 16-18

\section{Process sustainability evaluation}

To assist design engineers a tool has been developed to assess impacts to a nascent process in the form of the Sustainability Evaluator. This new Microsoft Excel-base$\mathrm{d}$ tool employs inputs of energy and mass for sustainability evaluation. This tool applies selected metrics that address economic, environmental, and health and safety concerns. This impact assessment tool provides the process designer with a framework to design industrial processes for sustainability. Process designers are expected to use the results generated from the tool to assess and improve the sustainability of a process. Every industrial process is assumed to be designed to maximize profits. Without economic viability a process is not sustainable. The tool organizes a selected group of sustainability metrics as economic, environmental, and social indices in the input logic of the evaluator.

The Sustainability Evaluator was evaluated for performance by analyzing two options for the synthesis of dimethyl ether (DME). Aspen Plus was used to simulate the test case model. Process comparison offered the opportunity for selection of the most sustainable option. The two synthetic routes are dehydration of $\mathrm{CH}_{3} \mathrm{OH}$ (methanol) and oxidation of $\mathrm{CH}_{4}$ (methane).

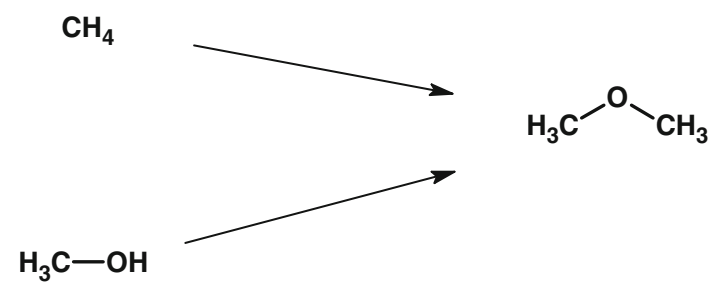

The methanol-based process was found to be the more sustainable DME production process. The environmental impact assessment for the methanol process showed that in terms of global warming, photochemical smog, and aquatic oxygen demand that the methane-based process was less environmental friendly. The authors hope that the tool will assist the design of economically and socially acceptable chemical processes thereby reduce negative impacts to the ecosystem.

Environ Prog Sus Energy 2013, 32, 749-761

\section{Climate change and disaster risk integration into development strategy}

A 58 page report produced collaboratively by the World Bank's Climate Finance and Policy team and the Global Facility for Disaster Reduction and Recovery investigates the integration of climate and disaster risk into development. All countries are susceptible to the adverse consequences of weather. Economic growth and markets are vulnerable to weather-related events. The effect of weather can be managed through good foresighted preparation to mute the unconstrained effects of crippling weather events.

Severe floods, droughts and storms, attributable from climate change, are expected to lead to increasing losses in the developing world for the near term future. Some 325 million people ensconced in poverty and vulnerable to weather-related events in sub-Saharan Africa and South Asia are among the susceptible populations across the globe. The poor and poor countries are expected to face escalating losses. Even middle-income nations are not exempt to the effects of climate change induced weather effects. For instance such nations could encounter combined annual losses in excess of \$1 trillion (US) in large coastal cities by 2050 as a result of these detrimental weather effects. This report shows climate resilience to be a critical element of World Bank goals to end extreme poverty and build shared prosperity. The report is expected to contribute to international dialog dedicated to 
developing an understanding of loss and damage from climate change. Without better adaptation to climate and disaster risks, the authors anticipate decades of development progress reversals. The international development community is enlisted to build long-term resilience, reduce risk, and avoid climbing future costs. Where resilience is effective, a higher initial investment is often required. Construction of safer buildings and infrastructure after a disaster often shows cost escalations of $50 \%$ for design and building. Enhanced weather warning systems designed to assist the resilience effort also require new technology and trained analysts. Relocation costs are expensive and may be associated with cultural and social disruptions. Strong social bonds within communities assist the community resilience to disaster. With greater attentive preparations, climate-related disasters are more manageable with reduced impacts and investment costs. No single discipline is able to meet the range of needs implied in the desired response to make climate and disaster resilience central to our day-to-day development work.

The report calls for the nations of the world to join in a collaborative effort to address disasters and help build resilience in the face of a rapidly warming world.

http://www.worldbank.org/content/dam/Worldbank/document/ SDN/Full_Report_Building_Resilience_Integrating_Climate_ Disaster_Risk_Development.pdf

\section{Extreme weather risks for the global insurance market}

A remarkable 38 page report: Warming of the Oceans and Implications for the (Re)insurance Industry, produced by the Geneva Association, reports on the evidence for ocean warming and its risk effects for the insurance industry. Robust evidence is available which shows that the global oceans have warmed significantly. Ocean energy is a key component of extreme events. Insurance-relevant hazards have increased to a new normal as attributed to ocean warming according to many climate- and weather-based indices. These changes are termed "quasi irreversible" since oceanic temperatures will continue to rise despite complete reduction of greenhouse gas (GHG) emissions. The report calls for a paradigm shift to predictive risk assessment methods using past historical data analysis which is necessary to estimate current hazardous probabilities. Current likelihood of extreme events in a nonstationary environment is severely limited due to the predictability and scientific understanding of extreme events. Scenario-based approaches and tail-risk modeling become essential parts of risk management. For certain high-risk areas, ocean warming and climate change threaten the insurability of catastrophe risk more generally. To avoid market failure, the coupling of risk transfer and risk mitigation becomes essential. The global ocean and its currents are of critical importance to the distribution and dissipation of solar energy absorbed in the global climate system. Energy is exchanged between the atmosphere and ocean mainly via radiative transfer and transport of latent heat from evaporation and condensation. Weather patterns are determined by the transportation of vast amounts of energy into the atmosphere. Extreme weather event are the result of weather patterns and energy exchange with the global ocean. Ocean dynamics and complex interactions between the atmosphere and ocean are implicit to understanding of distribution, frequency, and intensity of global extreme events, The insurance industry must be able to use relevant metrics to properly address events such as tropical cyclones, flash floods, or extra-tropical winter storms. Risk estimation related to these events must include recognition of the consequences due to the observed ocean warming.

The Geneva Association is an international insurance research group focused on the issues of insurance and risk management.

https://www.genevaassociation.org/media/616661/GA2013Warming_of_the_Oceans.pdf

\section{Microfluidic reactor technology}

During the past two decades, development of microfluidic systems for use in the chemical and biological sciences has shown extensive progress. This review assesses the function of microfluidic reactor technology in chemical synthesis as a tool for academic research and industrial applications. Advantages of small reagent volumes, selectivity, rapid reactions, small footprints, safe reactions, and green credentials offer a synthetic system important to a wide range of applications. Success and failure of the past research catalog of chemistries performed in a microfluidic reactor are scrutinized. Current obstacles to widespread use of microfluidic reactors from synthetic chemistry and industrial application perspective are assessed. The application of microfluidic technology permits the manipulation and control in geometrically constrained environments having internal dimensions, or hydrodynamic diameters, on a scale of micrometers which is the operational world of microfluidic systems. Notable contributions from academia and industry have been reported but industrial applications have slowly developed. Scale-dependent processes of heat and mass transfer are among the intrinsic advantages found with chemistry in microfluidic devices. Regimes of low Reynolds number are the norm in small fluidic volumes, where fluids are gradually influenced by viscosity rather than inertia. Thermal homogeneity throughout the reactor is enhanced by 
large ratios of surface area to volume and rapid heat transfer between the device and the contained fluid. These basic properties give rise to the following broad advantages that have made the technology attractive (in principle) for chemical synthesis in both industry and academia. The authors provide a set of seven challenges to inspire research.

An example of this technology's utility can be found with conversion of $\mathrm{m}$-bromoanisole to $\mathrm{m}$-anisaldehyde: accelerated warming trajectory is becoming important and forms the major analysis of this report. This analysis reports the findings of extensive modeling of the cryosphere regions.

As the result of new modeling in the five major cryosphere regions as well as globally for health, crop impacts, and climate benefits are identified. Cryosphere benefits are detailed for each region. Cook stove changes, important in the developing world, have measurably affected black carbon emissions. How meth-

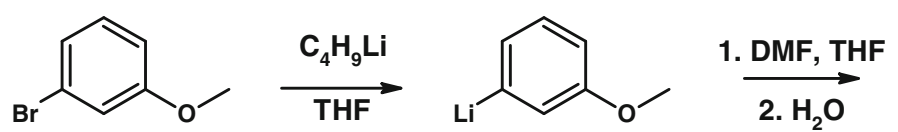

m-bromoanisole

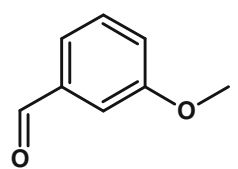

m-anisaldehyde
This two-step reaction is hard to scale up due to highly exothermic conditions and the reduction of yield as reactor size increases. It was possible to conduct the reaction in commercially available microscale reactors in contrast to the conventional macroscale, semicontinuous, batch reactors. The microscale reactors allowed the reaction to proceed without cryogenic cooling at higher temperatures due to the heat-exchange features of the microreactors.

Future challenges that were identified are compatible analytical technology, full use of surfaces, solids processing, mapping reaction space, fully selective multiproduct synthesis, truly different phases, and discovery chemistry. Microfluidic reaction systems have evolved into components of conventional synthetic and discovery laboratories. This review offers an overview of the application of microfluidic reactor technology with a recognition of success and failure applications as encountered at all scales. Supplemental information accompanying the paper provides the extensive and well-organized database used for the paper's analysis.

Nat Chem 2013, 5, 905-915

\section{Slowing global warming through pollution reduction}

A 116 page World Bank report: On thin ice: how cutting pollution can slow warming and save lives concentrates on the environmental effects of pollution to ice-covered regions of the globe. Climate change occurs faster and is more dramatically visible in the Earth's cryosphere than elsewhere on earth. Cryosphere is defined as elements of the Earth system containing water in its frozen state. The average temperature in the Arctic, Antarctic Peninsula, and much of the Himalayas and other mountain regions has risen more than twice the global mean. For the five major cryosphere regions: the Andes, Antarctica, Arctic, East African Highlands, and the Himalayas an ane and black carbon impact climate specifically in cryosphere regions and how the pollutants affect vulnerable populations continues to develop. Implications and new directions for the cryosphere regions found in these modeling results are discussed. The report is aimed at the information needs of decisionmakers and other stakeholders to provide an assessment of the cryosphere and how changes can affect human populations.

http://documents.worldbank.org/curated/en/2013/10/18496 924/thin-ice-cutting-pollution-can-slow-warming-save-livesvol-1-2-main-report

\section{Search for one-pot aniline production process}

Catalyst development and engineering strategies for the onepot transformation of benzene to aniline are the focus of this review. Aniline is commercially available from a variety of current industrial processes but there are significant economic and environmental issues related to each. For the current industrial processes, the nature of the catalysts, catalysts preparations and characterizations, performance and optimization options are scrutinized for aniline yield, selectivity, nature of the amination agents, and nature of oxidation agents. The M/MO cataloreactants, metal redox catalysts, and mesoporous crystalline materials receive special attention as the basis of new replacement technologies.
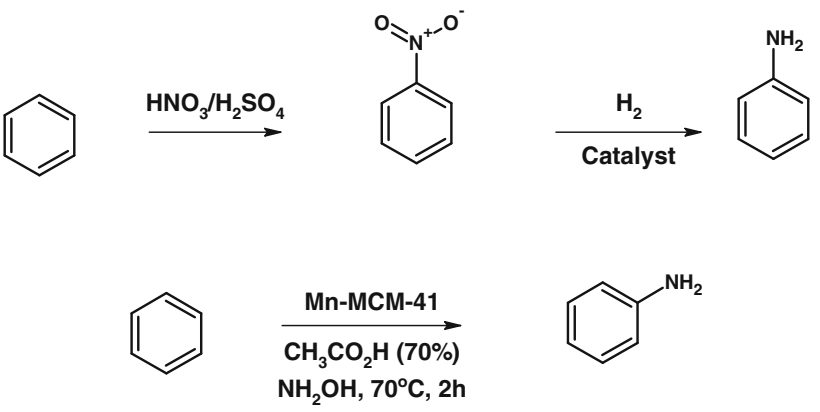
Academic and industrial research interests are expected to drive the one-pot search into the future using and modifying established technology. Nanomaterials may find a role in this search for sustainable technology.

Crit Revs Environ Sci Technol 2013, 43, 84-120

\section{Nanopesticide environmental impacts}

Nanopesticides have been the development target for a host of commercial sector research. More than 3,000 patent applications have been filed. Plant protection products are slowly being released to the markets. Corresponding extensive developments in pharmaceutical and personal care in the form of nanoformulations are already in use. Nanotechnology has been targeted for its potential to revolutionize agricultural practice. Formulations containing materials in the nanoparticle size range or properties associated with this particle size range have been available in the market for several years. The definition of nanopesticide varies widely in terms of particle

\section{Green process design simplified}

The Kolbe-Schmitt synthesis of 2,4-dihydroxybenzoic acid from resorcinol was used to investigate the utility of process intensification, and various reaction conditions in search of an environmentally benign process. A range of $\mathrm{CO}_{2}$ precursors [aqueous potassium hydrogen carbonate, hydrogen carbonate-based ionic liquids, DIMCARB (dimethylamine: $\mathrm{CO}_{2}$, or $\mathrm{SC}-\mathrm{CO}_{2}$ ] with the application of microwave irradiation for fast volumetric heating of the reaction mixture were investigated. Experiments were conducted in conventional batch-wise conditions and microstructured reactors to explore reaction conditions and aimed at the development of an environmentally benign process for the preparation of 2,4-dihydroxybenzoic acid. Simplified life cycle assessment (SLCA) was conducted for each process route. Convective-heating methods such as oil bath or electrical heating were found to be more beneficial than the application of microwave irradiation. Workup procedures were crucial for a complete view on the environmental burdens of each process route.

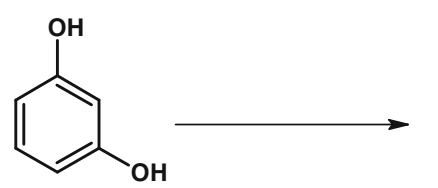<smiles>O=C(O)c1ccc(O)cc1O</smiles>

2,4-dihydroxybenzoic acid<smiles>O=C(O)c1c(O)cccc1O</smiles>

2,6-dihydroxybenzoic acid size range and nanoproperties claimed. Nanopesticide formulations are designed to assist the solubility of poorly soluble active ingredients and/or exert control over the release of the active ingredient. Different formulations (microemulsion, nanoemulsion, and nano dispersion) are designed to assist the solubilization of nanoparticle materials and provide different levels of emulsion/dispersion stability for a broad range of particle sizes. The environmental fate and effects for these new pesticide formulations are largely unknown. Environmental fates of these new articles of commerce will depend on particle size and ancillary constituents of the emulsion/dispersion. Flowing from these considerations are the concerns relating to the human-health and ecotoxicological features of nanopesticides. With the invention of any new plant protection agents, there is always important environmental and sustainability considerations of toxicity integral to the use of a new protection tool.

Crit Rev Environ Sci Technol 2013, 43, 1823-1867
The elimination of the side product (2,6-dihydroxybenzoic acid) was used as the production issue. Of the process options investigated, none could satisfy sustainability and production issues. No clear advantages were found to distinguish batch mode operations from the microstructured reactors. The aqueous potassium hydrogen carbonate conditions exhibited the lowest global warming potential (GWP) but low selective production of the desired product. Workup procedures were investigated for contribution GWP. Workup of the aqueous Kolbe-Schmitt showed an increase in GWP.

Environ Sci Technol 2013, 47, 5362-5371; Chem Eng J 2012, 200-202, 738-747

\section{New journal}

A new journal Biotechnology Reports begins publication in 2014 covering ground-breaking science in all aspects of biotechnology including work useful and informative to 
researchers in related fields. The journal seeks contributions to maintain high quality peer reviews of submissions exhibiting strong characteristics of scientific validity and technical quality. Research articles (short or full communications), methods, mini-reviews, and commentaries in topic areas to be considered are: healthcare and pharmaceutical applications, agricultural and food inventions, environmental biotechnology, molecular biology, cell and tissue engineering and synthetic biology, industrial biotechnology with biofuels and bioenergy, nanobiotechnology, bioinformatics \& systems biology, and new processes and products in biotechnology, bioprocess engineering.

http://www.journals.elsevier.com/biotechnology-reports/ 